\title{
Study of miR-21 \& MMP-3 Expression in Metastatic Breast Cancer Cell Line after Suppression of Bach 1 Gene Expression by a Small Interference RNA (siRNA)
}

\author{
Ali Aghebati-Maleki ${ }^{1}$, Leila Mohamed-Khosroshahi ${ }^{1,3}$, leili Aghebati- \\ Maleki ${ }^{1,2,3, *}$ \\ ${ }^{1}$ Immunology Research Center, Tabriz University of Medical Sciences, Tabriz, Iran \\ ${ }^{2}$ Department of Immunology, School of Medicine, Tabriz University of Medical \\ Sciences, Tabriz, Iran \\ ${ }^{3}$ Student Research Committee, Tabriz University of Medical Sciences, Tabriz, Iran \\ ${ }^{*}$ Corresponding author: Leili agehbati-Maleki; Student Research Committee, Tabriz \\ University of Medical Sciences, Tabriz, Iran. Tel: +4133364665-98, Fax: +-98 \\ 413364665, E-mail:aghebatil@tbzmed.ac.ir.
}

DOI: $10.21859 / \mathrm{mci}-$ supp-74

Keywords:

bach1

siRNA

miR-21

MMP-3

RNA Interference

Breast Cancer

\begin{abstract}
Introduction: Breast cancer is one of the common cancers especially among women that occurs all over the world. In spite of clinical and therapeutic progressions in recent years, many of patients also lose their lives after operation and metastasis. Bach1 gene plays a major role in growth of cancer, invasion, metastasis and inhibition of apoptosis in tumor cells. The purpose of this study is to knockdown bach1 gene and survey its effect on expression level of miR-21 and MMP-3.

Materials and Methods: In this study which is an experimental study, after designing and making of specific siRNA of the bach1 gene, its transfect on MDA-MB-468 cell lines was applied. Expression level of the gene analyzed by qRT-PCR. Proliferation of the cells examined by means of trypan blue experiment. Metastasis and cell invasion applied by examining of MMP-3 expression. At the end, expression level of miR-21 examined too.

Results: A significant decrease in gene expression of bach 1 in effective dosage $(p=0.001)$, metastasis and cell invasion was observed and also resulted of bach 1 knockdown in a significant decrease in expression of mir-21 ( $\mathrm{P}=0.0014)$ and MMP-3 $(\mathrm{P}<0.0001)$.

Conclusions: Bach1 knockdown can play a role in decreasing of breast cancer's impacts particularly in metastasis and its invasion besides, it makes decrease in the expression of metastatic microRNA such as miR-21. Therefore, knockdown of bach1 gene can have an effective therapeutic impact on breast cancer. Bach1 and miR-21 are considered as a diagnostic marker in breast cancer.
\end{abstract}

